\title{
Absolute continuity of the solution to the stochastic Burgers equation
}

\author{
Christian Olivera ${ }^{1, *}$ Ciprian A. Tudor ${ }^{2 \dagger}$ \\ 1 Departamento de Matemática, Universidade Estadual de Campinas, \\ 13.081-970-Campinas-SP-Brazil. \\ colivera@ime.unicamp.br \\ ${ }^{2}$ Université de Lille, CNRS \\ Laboratoire Paul Painlevé UMR 8524 \\ F-59655 Villeneuve d'Ascq, France. \\ tudor@math.univ-lille1.fr
}

March 8, 2021

\begin{abstract}
We prove the existence and the Besov regularity of the density of the solution to a general parabolic SPDE which includes the stochastic Burgers equation on an unbounded domain. We use an elementary approach based on the fractional integration by parts.
\end{abstract}

MSC 2010: Primary 60H15: Secondary 60H05.

Key Words and Phrases: stochastic Burgersr equation, space-time white noise, existence of the density, Besov spaces, fractional integration by parts.

\section{Introduction}

We consider the following parabolic stochastic partial differential equation (SPDE in the sequel)

$$
\frac{\partial}{\partial t} u(t, x)=\frac{\partial^{2}}{\partial x^{2}} u(t, x)-\frac{\partial}{\partial x} g(u(t, x))+\sigma(t, x, u(t, x)) \frac{\partial^{2} W}{\partial t \partial x}
$$

\footnotetext{
${ }^{*}$ C. O. is supported by FAPESP by the grant 2020/04426-6 , by CNPq by the grant $426747 / 2018-6$ and CAPES by the grant 88887.198637/2018-00- MATHAMSUD project SARC (19-MATH-06).

${ }^{\dagger}$ C. T. acknowledges partial support from the Labex CEMPI (ANR-11- LABX-0007-01) and MATHAMSUD project SARC (19-MATH-06).
} 
where the random noise $W$ is a Brownian sheet and the initial condition $u_{0}$ is deterministic. Our purpose is to cover both the situations when the space variable belongs to the whole real line and to the unit interval $[0,1]$. That is, will assume in (11) that

$$
(t, x) \in[0, T] \times I \text { with } I=\mathbb{R} \text { or } I=[0,1] .
$$

In both situations, we will assume

$$
u_{0}(t, x)=u_{0}(x) \text { for every } t \in[0, T], x \in I
$$

while for $I=[0,1]$ we also impose Dirichlet boundary conditions

$$
u_{0}(t, 0)=u_{0}(t, 1)=0 \text { for every } t \in[0, T] .
$$

In the particular case $g(x)=\frac{1}{2} x^{2}$, the equation (10) is called the stochastic Burgers equation and it has been studied by many authors. It is one of the most known singular stochastic partial differential equations. For a complete description of the deterministic Burgers equation (i.e. $\sigma \equiv 0$ in (10) we refer to the monograph [6] and the references therein. The Burgers equation with a random forcing term has been also studied by several authors, see e.g. [1], [4], [5], 7], 8], [1], 15], [14, [17], 18, [19], 20], 21]. The interest to study such stochastic equations comes from the fact during the past few decades, the stochastic Burgers equation has found applications in various fields ranging from statistical physics, cosmology, and fluid dynamics to engineering. In particular, the problem of Burgers turbulence, that is, the study of the solutions to the Burgers equation with random initial conditions or with a random noise is a central issue in the study of nonlinear systems out of equilibrium (see e.g. the surveys [3] and [12]).

Let us recall some known facts concerning the existence, the uniqueness and the properties of the solution to (11). For instance, in the case of the unbounded spatial domain, the existence and the pathwise properties of the solution have been studied in [4] (for the additive noise, i.e. $\sigma \equiv 1)$, [16], [19], while for the case of a bounded domain we refer to [19] (for the particular case of the stochastic Burgers equation $g(x)=\frac{1}{2} x^{2}$ ) or [15], 21] for a more general situation.

Our purpose is to discuss the absolute continuity of the law of the solution to (1) with a particular focus on the case of the Burgers equation. The existence and some regularity of the density of the solution are already known in the case of a bounded domain. Indeed, such results have been obtained in [19] or [21] by using the techniques of the Malliavin calculus, which requires rather stronger assumptions on the coefficients and technical proofs. As far as we know, there are no results concerning the absolute continuity of the law of the solution when the space variable belongs to the whole real line.

In order to prove the existence of a density for the solution to the parabolic SPDE (11), we propose here a different approach, based on the fractional integration by parts technique developed in [23] or [10]. This method is rather simple, it fits well with some classes of stochastic (partial) differential equations and it allows to obtain some properties of the density that cannot be obtained via Malliavin calculus or other techniques. Examples 
of applications of the integration by parts technique to the study of densities of solutions to various types of stochastic equations can be found in [9], [10, [13], 23], [24, [22. We obtain via a simple application of this method the existences and the Besov regularity of the density of the solution to (10), including the case of the Burgers equation $g(x)=\frac{1}{2} x^{2}$. As we pointed out before, such a result seems to be completely new for the unbounded domain while it provides a simpler alternative to the more technical proofs in [19] or [21] in the case $I=[0,1]$.

We organized our work as follows. Section 2 includes some general facts on the parabolic SPDEs of type (11), on its associated fundamental solution (Green kernel) and the integration by parts (or "smoothing" lemma). In Section 3 we apply the fractional integration by parts technique to get the existence and the regularity in Besov dense for the density of the solution to (1) while in Section 4 we discuss several examples, including the case of the Burgers equation on the real line.

By $c, C$ we denote generic strictly positive constants that are allowed to change from one line to another (and even on the same line).

\section{Preliminaries}

In this preliminary part, we define the noise and the solution to the parabolic SPDE (1). We also recall the fractionla integration by parts lemma which is the key element to obtain the existence and the Besov regularity of the density.

\subsection{The equation}

Let $I=[0,1]$ or $I=\mathbb{R}$ and let $(W(t, x), t \in[0, T], x \in I)$ be a centered Gaussian field defined on a complete probability space $(\Omega, \mathcal{F}, P)$ with covariance, for every $s, t \in[0, T]$ and $x, y \in I$

$$
\mathbf{E} W(s, x) W(t, y)=(s \wedge t)(|x| \wedge|y|) 1_{(0, \infty)}(x y) \text { if } I=\mathbb{R}
$$

and

$$
\mathbf{E} W(s, x) W(t, y)=(s \wedge t)(x \wedge y) \text { if } I=[0,1] .
$$

We will denote by $\mathcal{F}_{t}$ the sigma-algebra generated by the random variables $(W(s, x), 0 \leq s \leq t, x \in I)$. We assume that $\mathcal{F}=\mathcal{F}_{T}$ and we will say that a process $(Y(t, x), t \in[0, T], x \in I)$ is adapted with respect to the filtration $\left(\mathcal{F}_{t}\right)_{t \in[0, T]}$ if the random variable $Y(t, x)$ is $\mathcal{F}_{t}$-measurable for every $t \in[0, T], x \in I$.

The solution to (1) is understood in the mild sense, that is $u$ is a $\mathcal{F}_{t^{-}}$-adapted stochastic field that satisfies the integral equation

$$
\begin{aligned}
u(t, x)= & \int_{I} G_{t}(x-y) u_{0}(y) d y+\int_{0}^{t} \int_{I} \frac{\partial}{\partial y} G_{t-s}(x-y) g(u(s, y)) d y d s \\
& +\int_{0}^{t} \int_{I} G_{t-s}(x-y) \sigma(u(y, u(s, y))) W(d s, d y), \quad(t, x) \in[0, T] \times I .
\end{aligned}
$$


where the stochastic integral $W(d s, d y)$ is the Walsh integral with respect to Brownian sheet $W$ (see [26] for its definition and properties) and the Green kernel $G$ is the fundamental solution associated to the heat equation. It has the following expression (upon the situations $I=\mathbb{R}$ or $I=[0,1])$ : for $t>0$,

$$
G_{t}(x):=G_{1, t}(x)=\frac{1}{\sqrt{4 \pi t}} e^{-\frac{x^{2}}{4 t}} \text { if } I=\mathbb{R}
$$

and

$$
G_{t}(x):=G_{2, t}(x)=\frac{1}{\sqrt{4 \pi t}} \sum_{n=-\infty}^{\infty}\left[e^{-\frac{(y-x-2 n)^{2}}{4 t}}-e^{-\frac{(y+x-2 n)^{2}}{4 t}}\right] \text { if } I=[0,1] .
$$

The following properties of the Green kernel will be needed later.

Lemma 1 Let $G_{1}, G_{2}$ be given by (3), (4) respectively. Then we have

1. For every $0<\varepsilon<t$, we have

$$
\int_{t-\varepsilon}^{t} \int_{\mathbb{R}} G_{1, t-s}(x-y) d y d s \geq C_{2} \sqrt{\varepsilon} \text { and } \int_{t-\varepsilon}^{t} \int_{0}^{1} G_{2, t-s}(x-y) d y d s \geq C \sqrt{\varepsilon} .
$$

2. For every $x \in I$ and $t \in(0, T]$,

$$
\int_{\mathbb{R}} G_{1, t}^{2}(x-y) d y \leq C t^{-\frac{1}{2}} \text { and } \int_{\mathbb{R}} G_{2, t}^{2}(x-y) d y \leq C t^{-\frac{1}{2}}
$$

3. For every $x \in I$ and $t \in(0, T]$,

$$
\int_{\mathbb{R}}\left|\frac{\partial}{\partial y} G_{1, t}(x-y)\right| d y \leq C t^{-\frac{1}{2}} \text { and } \int_{0}^{1}\left|\frac{\partial}{\partial y} G_{2, t}(x-y)\right| d y \leq C t^{-\frac{1}{2}}
$$

Proof: The proof of the lower bound of point 1 . is immediate for $I=\mathbb{R}$, since by (3)

$$
\begin{aligned}
& \int_{t-\varepsilon}^{t} \int_{I} G_{1, t-s}^{2}(x-y) d y d s=C \int_{t-\varepsilon}^{t}(t-s)^{-1} \int_{\mathbb{R}} e^{-\frac{(x-y)^{2}}{2(t-s)}} d y d s \\
= & C \int_{t-\varepsilon}^{t}(t-s)^{-\frac{1}{2}} d s=C \varepsilon^{\frac{1}{2}}
\end{aligned}
$$

while for $I=[0,1]$ and $G=G_{2}$, the inequality in the statement has been proved in Lemma A.2 in [21]. Concerning point 2., by relation (2.4) in [19], with some $a, b>0$,

$$
\left|G_{2, t}(x, y)\right| \leq a t^{-\frac{1}{2}} e^{-\frac{b|x-y|^{2}}{t}}
$$

and then

$$
\int_{0}^{1} G_{2, t}^{2}(x-y) d y \leq a^{2} t^{-1} \int_{\mathbb{R}} e^{-\frac{2 b|x-y|^{2}}{t}} d y \leq C t^{-\frac{1}{2}}
$$


The same calculations are obvious for $G_{1}$, since

$$
\int_{\mathbb{R}} G_{1, t}^{2}(x-y) d y=C t^{-1} \int_{\mathbb{R}} e^{-\frac{|x-y|^{2}}{2 t}} d y \leq C t^{-\frac{1}{2}} .
$$

For point 3., when $I=[0,1]$ we have by relation (2.5) in [19], for some $a, b>0$, abnd for every $x, y \in I, t \in(0, T]$,

$$
\left|\frac{\partial}{\partial y} G_{2, t-s}(x-y)\right| \leq a t^{-1} e^{-\frac{b|x-y|^{2}}{t}}
$$

and thus

$$
\int_{0}^{1}\left|\frac{\partial}{\partial y} G_{2, t-s}(x-y)\right| d y \leq C t^{-1} \int_{\mathbb{R}} e^{-\frac{b|x-y|^{2}}{t}} d y \leq C t^{-\frac{1}{2}} .
$$

When $I=\mathbb{R}$, the above two relations also also true for the kernel $G_{1}$.

The existence and the uniqueness of the solution has been obtained in e.g. [4], 15], [16]. The conditions for the existence and uniqueness are slightly different in the cases of the bounded or unbounded domain. In order to kep an unitary approach, we will first show the absolute continuity of the law of (2) by assuming the existence and the pathwise regularity of the solution. In the last part of the paper which contains the examples, we will describe for each case the conditions that ensure existence, uniqueness and other properties of the solution.

\subsection{Besov spaces and the fractional integration by parts}

The monograph [25] offers a complete exposition on Besov spaces. In this work we only need to consider the particular Besov space $\mathcal{B}_{1, \infty}^{s}$ with $s>0$, which is defined below.

Consider a function $f: \mathbb{R} \rightarrow \mathbb{R}$ and for every $x, h \in \mathbb{R}$, put $\left(\Delta_{h}^{1} f\right)(x)=f(x+h)-f(x)$ and for $n \geq 1$ integer, define the $n$th increment of the function $f$ at lag $h$ by

$$
\left.\left(\Delta_{h}^{n} f\right)(x)=\Delta_{h}^{1}\left(\Delta_{h}^{n-1} f\right)\right)(x)=\sum_{j=0}^{n}(-1)^{n-j}\left(\begin{array}{l}
n \\
j
\end{array}\right) f(x+j h) .
$$

For instance, if $m=2,\left(\Delta_{h}^{n} f\right)(x)=f(x+2 h)-2 f(x+h)+f(x)$. For $0<s<n$ we define the norm

$$
\|f\|_{\mathcal{B}_{1, \infty}^{s}}=\|f\|_{L^{1}(\mathbb{R})}+\sup _{|h| \leq 1}|h|^{-s}\left\|\Delta_{h}^{n} f\right\|_{L^{1}\left(\mathbb{R}^{d}\right)} .
$$

It can be shown that for any $n, m>s$, the norms obtained in (6) using different $n$ and $m$ are equivalent. Therefore, one can define the Besov space $\mathcal{B}_{1, \infty}^{s}$ as the set of functions $f \in L^{1}(\mathbb{R})$ such that $\|f\|_{\mathcal{B}_{1, \infty}^{s}}<\infty$.

For $\alpha \in(0,1)$, let $C_{b}^{\alpha}(I)$ denote the set of bounded measurable functions $h: I \rightarrow \mathbb{R}$ such that

$$
\|h\|_{C_{b}^{\alpha}(I)}:=\|h\|_{\infty}+\sup _{x, y \in I, x \neq y,|x-y| \leq 1} \frac{|h(x)-h(y)|}{|x-y|^{\alpha}}<\infty .
$$


Our main tool to get the existence and the regularity of the density of the solution to (11) is the following smoothing lemma from [23]. (also called the fractional integration by parts lemma).

Lemma 2 Let $X$ be a real valued random variable. If there exist an integer $m \geq 1$, two real numbers $s>0, \gamma>0$, with $\gamma<s<m$, and a constant $K>0$ such that for every $\phi \in C_{b}^{\gamma}(\mathbb{R})$ and $h \in \mathbb{R}$, with $|h| \leq 1$,

$$
\mathbf{E}\left[\Delta_{h}^{m} \phi(X)\right] \leq K|h|^{s}\|\phi\|_{C_{b}^{\gamma}(\mathbb{R})},
$$

then $X$ admits a density $f_{X}$ with respect to Lebesgue measure on $\mathbb{R}$. Moreover, $f_{X} \in B_{1, \infty}^{s-\alpha}$ and $\|f\|_{B_{1, \infty}^{s-\alpha}} \leq C(1+K)$.

\section{The existence and Besov regularity of the density}

We will use the fractional integration by parts (Lemma 21) in order to obtain the absolute continuity of the law of the random variable $u(t, x)$ given by (2), for fixed $t \in(0, T]$ and $x \in I$. We will state and prove a general result, that includes both the cases of bounded and unbounded domains, by assuming the existence, the Hölder regularity in time and some moment estimates for the solution to (11). In the next section, we will treat separately several particular cases and for each of them we will show that these properties are satisfied.

Let us consider the following assumptions: there exist $L_{1}, L_{2}, K>0$ such that

- The diffusion coefficient $\sigma: I \times \mathbb{R} \rightarrow \mathbb{R}$ does not depend on time and it is globally Lipschitz i.e.

$$
|\sigma(x, r)-\sigma(x, v)| \leq L_{1}|r-v| \text { for every } r, v \in \mathbb{R}, x \in I .
$$

and

$$
|\sigma(x, r)|^{2} \geq K \text { for every } x \in I, r \in \mathbb{R} .
$$

- The function $g: \mathbb{R} \rightarrow \mathbb{R}$ satisfies

$$
|g(r)-g(v)| \leq L_{2}(1+|r|+|v|)|r-v| \text { for every } r, v \in \mathbb{R} .
$$

We assume for simplicity that $\sigma$ does not depend on time. The case of a timedepedent diffusion coefficient can be treated in a similar way, by assuming the Lipschitz condition in time for it. Notice that the above conditions (7), (8) and (9) does not guarantee the existence of the solution (2), see Section 4.

Let us state our general result concerning the absolute continuity of the law of the solution to (1). 
Theorem 1 Assume (7), (8) and (9). Also assume that the equation (1) admits a unique solution $(u(t, x), t \in[0, T], x \in I)$ and there exists $\beta>0$ such that for every $p \geq 2$,

$$
\mathbf{E}|u(t, x)-u(s, x)|^{p} \leq c|t-s|^{\beta p}
$$

for every $x \in I, t, s \in[0, T]$ and

$$
\sup _{t \in[0, T], x \in I} \mathbf{E}|u(t, x)|^{p} \leq C_{T}
$$

Then for every $t \in(0, T]$ and $x \in I$, the random variable $u(t, x)$ admits a density which belongs to the Besov space $\mathcal{B}_{1, \infty}^{p}$ with $p<2 \beta$.

Proof: Fix $t \in(0, T]$ and $0<\varepsilon<\frac{t}{2}$. We introduce the auxiliary processes

$$
\begin{aligned}
u_{0, \varepsilon}(t, x)= & \int_{I} G_{t}(x-y) u_{0}(y) d y+\int_{0}^{t-\varepsilon} \int_{\mathbb{I}} \frac{\partial}{\partial y} G_{t-s}(x-y) g(u(s, y)) d y d s \\
& +\int_{0}^{t-\varepsilon} \int_{I} G_{t-s}(x-y) \sigma(u(y, u(s, y))) W(d s, d y)
\end{aligned}
$$

and

$$
\begin{aligned}
u_{\varepsilon}(t, x)= & u_{0, \varepsilon}(t, x)+\int_{t-\varepsilon}^{t} \int_{I} \frac{\partial}{\partial y} G_{t-s}(x-y) g(u(t-\varepsilon, y)) d y d s \\
& +\int_{t-\varepsilon}^{t} \int_{I} G_{t-s}(x-y) \sigma(u(y, u(t-\varepsilon), y)) W(d s, d y) .
\end{aligned}
$$

In order to apply Lemma 2, we need to calculate

$$
\mathbf{E}\left(\Delta_{h}^{m} \varphi\right)(u(t, x))
$$

for every $h>0, m \geq 1$ integer and $\varphi: \mathbb{R} \rightarrow \mathbb{R}$ a function in $C_{b}^{\gamma}(\mathbb{R})$ for $\gamma \in(0,1)$. We write

$$
\begin{aligned}
\mathbf{E}\left(\Delta_{h}^{m} \varphi\right)(u(t, x))= & \mathbf{E}\left(\Delta_{h}^{m} \varphi\right)\left(u_{\varepsilon}(t, x)\right)+\mathbf{E}\left[\left(\Delta_{h}^{m} \varphi\right)(u(t, x))-\left(\Delta_{h}^{m} \varphi\right)\left(u_{\varepsilon}(t, x)\right)\right] \\
=: & (P E)_{\varepsilon}(m, h, t, x)+(A E)_{\varepsilon}(m, h, t, x)
\end{aligned}
$$

with $u_{\varepsilon}$ given by (13). The first summand above is usually called the probabilistic estimate while the second term is the approximation error. Let us estimate separately these two quantities.

Calculation of the probabilistic estimate. Only condition (8) is needed for this estimate. We write, for every $t \in(0, T], x \in I$ and $0<\varepsilon<\frac{t}{2}$,

$$
u_{\varepsilon}(t, x)=Z_{t, x, \varepsilon}+I_{t, x, \varepsilon}
$$


with

$$
Z_{t, x, \varepsilon}=u_{0, \varepsilon}(t, x)+\int_{t-\varepsilon}^{t} \int_{I} \frac{\partial}{\partial y} G_{t-s}(x-y) g(u(t-\varepsilon, y)) d y d s
$$

and

$$
I_{t, x, \varepsilon}=\int_{t-\varepsilon}^{t} \int_{I} G_{t-s}(x-y) \sigma(u(y, u(t-\varepsilon), y)) W(d s, d y)
$$

From (14)

$$
\begin{aligned}
(P E)_{\varepsilon}(m, h, t, x) & =\mathbf{E}\left(\Delta_{h}^{m} \varphi\right)\left(u_{\varepsilon}(t, x)\right) \\
& =\mathbf{E}\left[\mathbf{E}\left(\left(\Delta_{h}^{m} \varphi\right)\left(u_{\varepsilon}(t, x)\right) / \mathcal{F}_{t-\varepsilon}\right)\right]=\mathbf{E}\left[\mathbf{E}\left(\left(\Delta_{h}^{m} \varphi\right)\left(Z_{t, x, \varepsilon}+I_{t, x, \varepsilon}\right) / \mathcal{F}_{t-\varepsilon}\right)\right] .
\end{aligned}
$$

Notice that the random variable $Z_{t, x, \varepsilon}$ given by (15) is measurable with respect to $\mathcal{F}_{t-\varepsilon}$ while $I_{t, x, \varepsilon}$ given by (16) is, conditionally on $\mathcal{F}_{t-\varepsilon}$, independent of $Z_{t, x, \varepsilon}$. Thus

$$
(P E)_{\varepsilon}(m, h, t, x)=\mathbf{E} f\left(Z_{t, x, \varepsilon}\right)
$$

with

$$
f(y)=\mathbf{E}\left[\left(\Delta_{h}^{m} \varphi\right)\left(y+I_{t, x, \varepsilon}\right)\right] \text { for every } y \in \mathbb{R} .
$$

Also, we can se that the random variable $I_{t, x, \varepsilon}$ is, conditionally on $\mathcal{F}_{t-\varepsilon}$, a Gaussian random variable with zero expectation and variance

$$
V_{t, x, \varepsilon}=\int_{t-\varepsilon}^{t} \int_{I} G_{t-s}^{2}(x-y) \sigma^{2}(y, u(t-\varepsilon, y)) d y d s
$$

So the conditional density of $I_{t, x, \varepsilon}$ is

$$
g_{t, x, \varepsilon}(z)=\frac{1}{\sqrt{2 \pi V_{t, x, \varepsilon}}} e^{-\frac{z^{2}}{2 V_{t, x, \varepsilon}}}, \quad z \in \mathbb{R} .
$$

Therefore

$f(y)=\int_{\mathbb{R}}\left(\Delta_{h}^{m} \varphi\right)(y+z) g_{t, x, \varepsilon}(z) d z=\int_{\mathbb{R}} \varphi(y+z)\left(\Delta_{-h}^{m} g_{t, \varepsilon}(z)\right) d z \leq\|\varphi\|_{\infty}\left\|\Delta_{-h}^{m} g_{t, \varepsilon}(z)\right\|_{L^{1}(\mathbb{R})}$.

We know from [23] (see page 5, two lines after (2.7)) that

$$
\left\|\Delta_{-h}^{m} g_{t, \varepsilon(x)}\right\|_{L^{1}(\mathbb{R})} \leq C\left(\frac{|h|}{\sqrt{V_{t, x, \varepsilon}}}\right)^{m} \leq C\left(\frac{|h|}{\varepsilon^{\frac{1}{2}}}\right)^{m}
$$

for every $h>0$ and for any integer $m \geq 1$. For the last inequality in (18) we used the fact that by assumption (8) and Lemma 1 point 1., for every $t, x, \varepsilon$,

$$
V_{t, x, \varepsilon} \geq K \int_{t-\varepsilon}^{t} \int_{I} G_{t-s}^{2}(x-y) d y d s \geq C \sqrt{\varepsilon} .
$$


Thus

$$
\sup _{y \in \mathbb{R}}|f(y)| \leq C\left(\frac{|h|}{\varepsilon^{\frac{1}{2}}}\right)^{m} .
$$

and (17) and (19) imply for every $h>0, m \geq 1$ integer and $(t, x) \in(0, T] \times I$,

$$
\left|(P E)_{\varepsilon}(m, h, t, x)\right| \leq C\left(\frac{|h|}{\varepsilon^{\frac{1}{2}}}\right)^{m} .
$$

Calculation of the approximation error. To handle the term $(A E)_{\varepsilon}(m, h, t, x)$ in (14), we will use the assumptions (7), (9), (10) and (11). Recall that by (14)

$$
(A E)_{\varepsilon}(m, h, t, x)=\mathbf{E}\left[\left(\Delta_{h}^{m} \varphi\right)(u(t, x))-\left(\Delta_{h}^{m} \varphi\right)\left(u_{\varepsilon}(t, x)\right)\right] .
$$

From (2) and (13), for every $0<\varepsilon<\frac{t}{2}$ and for every $x \in I$,

$$
\begin{aligned}
u(t, x)-u_{\varepsilon}(t, x)= & \int_{t-\varepsilon}^{t} \int_{I} \frac{\partial}{\partial y} G_{t-s}(x-y)(g(u(s, y))-g(u(t-\varepsilon, y))) d y d s \\
& +\int_{t-\varepsilon}^{t} \int_{I} G_{t-s}(x-y)(\sigma(u(y, u(s, y))-\sigma(y, u(t-\varepsilon, y))) W(d s, d y) .
\end{aligned}
$$

This implies, since $\gamma \in(0,1)$,

$$
\left|(A E)_{\varepsilon}(m, h, t, x)\right| \leq C\|\varphi\|_{C_{b}^{\gamma}(I)} \mathbf{E}\left|u(t, x)-u_{\varepsilon}(t, x)\right|^{\gamma} \leq C\|\varphi\|_{C_{b}^{\gamma}(I)}\left(\mathbf{E}\left|u(t, x)-u_{\varepsilon}(t, x)\right|\right)^{\gamma} .
$$

We then need to estimate $\mathbf{E}\left|u(t, x)-u_{\varepsilon}(t, x)\right|$. We can write,

$$
\begin{aligned}
\mathbf{E}\left|u(t, x)-u_{\varepsilon}(t, x)\right| \leq & \int_{t-\varepsilon}^{t} \int_{I}\left|\frac{\partial}{\partial y} G_{t-s}(x-y)\right| \mathbf{E}|g(u(s, y))-g(u(t-\varepsilon, y))| d y d s \\
& +\mathbf{E} \mid \int_{t-\varepsilon}^{t} \int_{I} G_{t-s}(x-y)(\sigma(u(y, u(s, y))-\sigma(y, u(t-\varepsilon, y))) W(d s, d y) \mid \\
:= & A+B .
\end{aligned}
$$

Now, by (9), (11) and Hölder's inequality

$$
\begin{aligned}
A & \leq C \int_{t-\varepsilon}^{t} \int_{I}\left|\frac{\partial}{\partial y} G_{t-s}(x-y)\right| \mathbf{E}(1+|u(s, y)|+|u(t-\varepsilon, y)|)|u(s, y)-u(t-\varepsilon, y)| d y d s \\
& \leq C \int_{t-\varepsilon}^{t} \int_{I}\left|\frac{\partial}{\partial y} G_{t-s}(x-y)\right|\left(\mathbf{E}|u(s, y)-u(t-\varepsilon, y)|^{2}\right)^{\frac{1}{2}}
\end{aligned}
$$

and by using the Hölder assumption (10) with $p=2$, we obtain 


$$
A \leq C \int_{t-\varepsilon}^{t} \int_{I}\left|\frac{\partial}{\partial y} G_{t-s}(x-y)\right|(s-t+\varepsilon)^{\beta} d y d s \leq C \varepsilon^{\beta} \int_{t-\varepsilon}^{t} \int_{I}\left|\frac{\partial}{\partial y} G_{t-s}(x-y)\right| d y d s
$$

By Lemma 1 point3. and using $0<\varepsilon<\frac{t}{2}$ with $t \in(0, T]$,

$$
A \leq C \varepsilon^{\beta} \int_{t-\varepsilon}^{t}(t-s)^{-\frac{1}{2}} d s \leq C \varepsilon^{\beta} \int_{t-\varepsilon}^{t} s^{-\frac{1}{2}} d s=C \varepsilon^{\beta}\left(t^{\frac{1}{2}}-(t-\varepsilon)^{\frac{1}{2}}\right) \leq C t^{-\frac{1}{2}} \varepsilon^{\beta+1} .
$$

For the term $B$, we have by (7D) and (10)

$$
\begin{aligned}
B^{2} & \leq \int_{t-\varepsilon}^{t} \int_{I} G_{t-s}^{2}(x-y) \mathbf{E}\left(\sigma(u(y, u(s, y))-\sigma(y, u(t-\varepsilon, y)))^{2} d y d s\right. \\
& \leq C \int_{t-\varepsilon}^{t} \int_{I} G_{t-s}^{2}(x-y) \mathbf{E}|u(s, y)-u(t-\varepsilon, y)|^{2} d y d s \\
& \leq C \int_{t-\varepsilon}^{t} \int_{I} G_{t-s}^{2}(x-y)(s-t+\varepsilon)^{2 \beta} d y d s \\
& \leq C \varepsilon^{2 \beta} \int_{t-\varepsilon}^{t}(t-s)^{-\frac{1}{2}} d y d s \leq C t^{-\frac{1}{2}} \varepsilon^{2 \beta+1}
\end{aligned}
$$

where we used Lemma 1 point 2. and we proceeded as for the bound (22). Therefore, by (21)

$$
\left|(A E)_{\varepsilon}(m, h, t, x)\right| \leq C\|\varphi\|_{C_{b}^{\gamma}(I)} \varepsilon^{\gamma\left(\beta+\frac{1}{2}\right)} .
$$

We obtained by (20) and (23), for every $h>0, m \geq 1$ integer and $(t, x) \in[0, T] \times I$,

$$
\left|\mathbf{E}\left(\Delta_{h}^{m} \varphi\right)(u(t, x))\right| \leq C\|\varphi\|_{C_{b}^{\gamma}(I)}\left[\varepsilon^{\left(\beta+\frac{1}{2}\right)}+\left(\frac{|h|}{\varepsilon^{\frac{1}{2}}}\right)^{m}\right]^{\gamma} .
$$

We choose $\varepsilon=h^{A}$ with suitable $A$ which optimizes the right-hand side of (24). This gives

$$
A=\frac{2 m}{m+2\left(\beta+\frac{1}{2}\right)}
$$

and so (24) becomes

$$
\left|\mathbf{E}\left(\Delta_{h}^{m} \varphi\right)(u(t, x))\right| \leq C\|\varphi\|_{C_{b}^{\alpha}(I)} h^{s} \text { with } s=\frac{2 m \gamma\left(\beta+\frac{1}{2}\right)}{m+2\left(\beta+\frac{1}{2}\right)} .
$$

When $m$ is large enough, we see that $s$ becomes arbirarly close to $2 \gamma\left(\beta+\frac{1}{2}\right)$. Then, by Lemma 2 , the solution to (11) admits a density and this density belongs to the Besov space $\mathcal{B}_{1, \infty}^{p}$ with

$$
p<s-\gamma=2 \gamma\left(\beta+\frac{1}{2}\right)-\gamma=2 \gamma \beta
$$


By choosing $\gamma$ arbitrarly close to 1 , we get that the density belongs to the Besov space $\mathcal{B}_{1, \infty}^{p}$ with $0<p<2 \beta$.

Notice that the Besov regularity of the density is related to the Hölder regularity in time of the solution. The spatial regularity (which is also known from [21] or [20]) of the solution does not affect the regularity of the density.

\section{Applications}

Let us now discuss the stochastic equation (11) in some particular cases.

\subsection{The stochastic Burgers equation on $\mathbb{R}$}

We will show that our result stated in Theorem 1 can be applied to the stochastic Burgers equation on the whole real line. Recall that no results are known in this case on the existence of the density of the solution.

We will assume throughout this section that

$$
I=\mathbb{R} \text { and } g(x)=\frac{1}{2} x^{2} \text { for every } x \in \mathbb{R}
$$

which corresponds to the case of the stochastic Burgers equation.

In order to ensure the existence of the solution (2) in the situation (25), let us consider the following additional conditions:

- For some $\alpha \in(0,1)$, the initial condition $u_{0}$ satisfies

$$
u_{0} \in L^{1}(\mathbb{R}) \cap L^{2}(\mathbb{R}) \cap C_{b}^{\alpha}(\mathbb{R}) .
$$

- The diffusion coefficient $\sigma$ is such that

$$
|\sigma(x, r)| \leq f(x) \text { for every } x, r \in \mathbb{R}
$$

where $f$ is a non-negative function such that $f \in L^{2}(\mathbb{R}) \cap L^{q}(\mathbb{R})$ with $q>2$.

The following result has been proved in [16] (the existence of the solution) and in [19] (the Hölder regularity and the moment estimates of the solution).

Theorem 2 Assume (7), (25), (26) and (27). Then (1) admits an unique solution which satisfies, for all $s, t \in[0, T], x, y \in \mathbb{R}$ and $p \geq 2$

$$
\mathbf{E}|u(t, x)-u(s, y)|^{p} \leq C\left(|t-s|^{\frac{\alpha}{2} \wedge\left(\frac{1}{4}-\frac{1}{2 q}\right)}+|x-y|^{\alpha \wedge\left(\frac{1}{2}-\frac{1}{q}\right)}\right)^{p}
$$

and

$$
\sup _{t \in[0, T], x \in \mathbb{R}} \mathbf{E}|u(t, x)|^{p} \leq C_{T} .
$$


We obtain, from the above result and Theorem 1 the absolute continuity of the the law of the stochastic Burgers equation on an unbounded domain.

Corollary 1 Assume (8), (7), (26), (27) and (25), and let $(u(t, x), t \in[0, T], x \in \mathbb{R})$ be the unique solution to (1). Then for every $t \in(0, T], x \in \mathbb{R}$, the random variable $u(t, x)$ admits a density (denoted $f_{t, x}$ ) which satisfies

$$
f_{t, x} \in \mathcal{B}_{1, \infty}^{p} \text { with } p<\alpha \wedge\left(\frac{1}{2}-\frac{1}{q}\right) \text {. }
$$

Remark 1 By choosing a regular enough initial condition (for example, $u_{0}$ is Hölder continuous or order $\alpha>\frac{1}{2}$ ), we obtain that the density of the solution to the Burgers equation belongs to the Besov space $\mathcal{B}_{1, \infty}^{p}$ with $p<\frac{1}{2}-\frac{1}{q}$ with $q$ from (27). The Hölder regularity in space of the solution does not affect the regularity of the density.

\subsection{The Burgers type equation on a bounded domain}

We will regard the density of the solution to (1) when the space variable belongs to the unit interval $[0,1]$. We will restrict to the case when the function $g$ in (11) is Lipschiz continuous. This is because, in order to apply Theorem 1 we need the Hölder continuity of the solution which has been proved, as far as we know, only for $g$ Lipschitz (see [21]).

Let us consider the following assumptions:

- For some $\alpha \in(0,1)$, the initial condition $u_{0}$ satisfies

$$
I=[0,1] \text { and } u_{0} \in C_{b}^{\alpha}(I) .
$$

- The function $g$ satisfies

$$
|g(x)-g(y)| \leq C|x-y| \text { for every } x, y \in[0,1] .
$$

The next result is Theorem 2.1 in [21].

Theorem 3 Assume (7), (8), (28) and (29). Then the stochastic equation (1) admits a unique solution which satisfies, for every $s, t \in[0, T], x, y \in[0,1]$ and $p \geq 2$

$$
\mathbf{E}|u(t, x)-u(s, y)|^{p} \leq C\left(|t-s|^{\frac{\alpha}{2} \wedge \frac{1}{4}}+|x-y|^{\alpha \wedge \frac{1}{2}}\right)^{p}
$$

and

$$
\sup _{t \in[0, T], x \in[0,1} \mathbf{E}|u(t, x)|^{p} \leq C_{T} .
$$

We can immediately conclude that our Theorem 1 can be applied. 
Corollary 2 Assume (7), (25), (26) and (27) and let $(u(t, x), t \in[0, T], x \in[0,1])$ be the unique solution to (1). Then for every $t \in(0, T], x \in \mathbb{R}$, the random variable $u(t, x)$ admits a density (denoted $f_{t, x}$ ) which satisfies

$$
f_{t, x} \in \mathcal{B}_{1, \infty}^{p} \text { with } p<\alpha \wedge \frac{1}{2}
$$

Notice that the above result also applies to the stochastic heat equation with space variable in $[0,1]$, by choosing $g \equiv 0$.

\subsection{The heat equation on $\mathbb{R}$}

Take $g \equiv 0$ and assume $I=\mathbb{R}$. The existence, the Hölder regularity and the moment estimates for the solution to (11) have been obtained in [26]. Actually, the relations (30) and (31) hold true in the case of the heat equation with spatial variable on the whole real line. The absolute continuity of the law of the solution is also well-known (see e.g. [2]). solution.

In this case, our result in Theorem 1 gives in addition the Besov regularity of the

Corollary 3 Let $g \equiv 0$ and $I=\mathbb{R}$ in (1) Assume (8), (7), (26), 27) and (25), and let $(u(t, x), t \in[0, T], x \in \mathbb{R})$ be the unique solution to (1)). Then for every $t \in(0, T], x \in \mathbb{R}$, the random variable $u(t, x)$ admits a density (denoted $f_{t, x}$ ) which satisfies

$$
f_{t, x} \in \mathcal{B}_{1, \infty}^{p} \text { with } p<p<\left(\alpha \wedge \frac{1}{2}\right) .
$$

We retrieve a result from [24], see Remark 3.4.

\section{References}

[1] S. Assing (2002): A pregenerator for Burgers equation forced by conservative noise. Commun. Math. Phys. 225, 611-632.

[2] V. Bally and E. Pardoux (1998): Malliavin calculus for white noise driven parabolic SPDEs. Potential Analysis, 9, 27-64.

[3] J. Bec and K. Khanin (2007): Burgers turbulence. Phys. Rep. 447, 1-66.

[4] L. Bertini, N. Cancrini and C. Jona-Lasinio (1994): The stochastic Burgers equation. Comm. Math. Phys. 165 (2), 211-232.

[5] Z. Brzezniak, B. Goldys and M. Neklyudov (2014): Multidimensional stochastic Burgers equation. SIAM J. Math. Anal. 46(1), 871-889.

[6] J. M. Burgers (1974): The nonlinear Diffusion Equation. Reidel, Dordrecht. 
[7] P. Catuogno and C. Olivera (2014): Strong solution of the stochastic Burgers equation. Appl. Anal. 93 (3), 646-652.

[8] G, Da Prato, A. Debussche, R. Temam (1994): Stochastic Burgers equation. NoDEA Nonlinear Differential Equations Appl. 1(4), 389-402.

[9] A. Debussche, N. Fournier (2013): Existence of densities for stable-like driven SDE's with Hölder continuous coefficients, Journal of Functional Analysis, 264 (8), 1757-1778.

[10] A. Debussche and M. Romito (2014): Existence of densities for the 3D Navier-Stokes equations driven by Gaussian noise, Probab. Theory Related Fields 158 (3-4), 575-596.

[11] A. Dermoune (1997): Around the stochastic Burgers equation. Stochastic Anal. Appl. 15(3), 295-311.

[12] W. E. (2000): Stochastic hydrodynamics. Current developments in mathematics, 109147, Int. Press, Somerville.

[13] N. Fournier and J. Printems (2010): Absolute continuity for some one-dimensional processes, Bernoulli 16 (2), 343-360.

[14] M. Gubinelli and N. Perkowski (2017): KPZ Reloaded, Communications in Mathematical Physics 349, 165-269.

[15] I. Gyongy (1998): Existence and uniqueness results for semilinear stochastic partial differential equations. Stochastic Process. Appl. 73 (2), 271-299.

[16] I. Gyongy, and D. Nualart (1999): On the stochastic Burgers' equation in the real line. Ann. Probab. 27, 2, 782-802.

[17] H. Holden, T. Lindstrom, B. Oksendal, J. Uboe and T. Zhang (1994): The Burgers equation with a noisy force and the stochastic heat equation. Comm. Partial Differential Equations, 19 (1-2), 119-141.

[18] J. U. Kim (2004): On the stochastic Burgers equation with a polynomial nonlinearity in the real line, Discrete Contin. Dyn. Syst. Ser. B, 6, 835-866.

[19] J.A Leon, D Nualart, R Pettersson (2000): The stochastic Burgers equation: finite moments and smoothness of the density. Infinite Dimensional Analysis, Quantum Probability and Related Topics, 3, 363-385.

[20] P. Lewis and D.Nualart (2018): Stochastic Burgers' equation on the real line: regularity and moment estimates. Stochastics 90, 1053-1086.

[21] P-L. Morien (1999): On the density for the solution of a Burgers-type SPDE. Ann. Inst. H. Poincaré Probab. Statist. 35 (4), 459-482. 
[22] C. Olivera and C.A. Tudor (2019): Existence and Besov regularity of the density for a class of SDEs with Volterra noise. C. R. Math. Acad. Sci. Paris 357 (7), 636-645.

[23] M. Romito (2018): A simple method for the existence of a density for stochastic evolutions with rough coefficients. Electronic Journal of Probability, 23, paper 113, 1-43.

[24] M. Sanz-Sole and Andre Süss (2015): Absolute continuity for SPDEs with irregular fundamental solution, Electron. Commun. Probab. 20 (14).

[25] H. Triebel (1983): Theory of function spaces. Monographs in Mathematics, 78, Birkhauser Velag, Basel..

[26] J. W Walsh (1986): An introduction to stochastic partial differential equations. École d été de probabilités de Saint-Flour, XIV-1984, 265-439, LNM 1180, Springer. 